\title{
Introduction to the Serious Games, Gamification and Innovation Minitrack
}

\author{
Imed Boughzala \\ Telecom Ecole de Management \\ Institut Mines-Telecom, Paris, France \\ imed.boughzala@it-sudparis.eu
}

\author{
Hélène Michel \\ Grenoble Ecole de Management \\ helene.michel@grenoble-em.com
}

Serious Games are often considered to be technological applications that use games to engage individuals in an experience through which a learning or professional training aim can be explored. Serious Games use computer-mediated environments to facilitate experiential learning by simulating a real life business environment, enabling participants to explore the complexities of a business problem, including the emotional challenges. A benefit is that the learner has the opportunity to explore the dynamics of a business experience without the real life business and career consequences that may flow from failure. This provides learners with a 'safe' environment in which to explore the casual links between action and outcomes that can assist in the process of converting conceptual learning into application.

In the innovation perspective, concepts such as serious gaming or gamification are the most interesting for this domain. Serious Gaming repurposes a game via different methods, in order to offer activities that go beyond mere entertainment and Gamification uses game design to enhance individual's willingness to participate to originally non-playful experiences (to improve user experience and user engagement).

Serious Games as learning methods have been widely developed since the 2000s mainly through a combination of major companies' projects or calls for tender and education initiatives by academics and academic publishers developing games for third level education programs. Many applications emerged in higher and continuing education, internal and external communication or public marketing. Today, new perspectives are explored in different dimensions of the organization: human resources management (through recruitment and assessment), knowledge management or innovation. Serious Games challenge the notions of prevention, training, knowledge assessment, coaching, communication, data collection, etc. in various fields such as education, healthcare, marketing, safety, culture to name but a few. In a decade, serious games became a field of research and business. Despite the growth in the use of serious games as an education and business development experiential learning tool, many management, education and development research questions remain underexplored and the literature remains disparate. Thus this minitrack covers Serious Games usage and gamification as new challenges of innovation.

This year, four papers were selected for inclusion in the proceedings. The first paper, "Feedback on the Integration of a Serious Game in the Data Modeling Learning" by Imed Boughzala, Olfa Chourabi, Daniel Lang and Mondher Feki, presents an exploratory experiment on the usage of the serious game Innov8, initially designed for learning BPM, through an application of gamification for learning data modeling to a business school students.

The second paper, "The Impact of Gamification on Word-of-Mouth Effectiveness: Evidence from Foursquare" by Lei Wang, Kunter Gunasti, Ram Gopal, Ramesh Shankar and Joseph Pancras, studies how gamification elements, which capture and signal contributors' accumulated expertise, affect consumers' perception of contributors' knowledge, and therefore the perceived effectiveness of their contributed WOM.

The third paper, "Exploring practical potentials of business simulation games" by J.B. Kim and Edward Watson, explores the business professional's sensemaking process when consciously reasoning about how BSG learning influences business practice.

The final paper, "Towards a Home-based Virtual Reality Game System to Promote Exercise" by Ashish Amresh and Rahul Salla, uses the design of the game mechanics that promote increased calorific output among the participants and the ease of setup with any commercially available Virtual Reality (VR) sensor.

The papers in this minitrack discuss different aspects related to the adaption of serious games and the use of gamification as an innovation. Each offers a unique contribution to our understanding of how serious games would be used to support learning. We commend them to your reading, and hope they will inspire your research and practice. 